\title{
Megacolon tóxico de origen idiopático: reporte de caso
}

\section{Megacolon toxic of idiophatic origin: case report}

Sergio Andrés Siado, ${ }^{1}$ Héctor Conrado Jiménez, ${ }^{2}$ Carlos Mauricio Martínez Montalvo. ${ }^{3}$

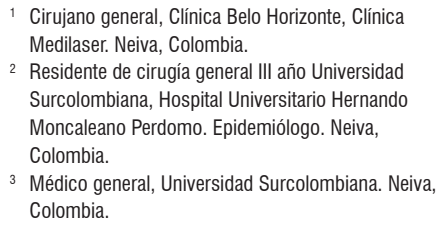

2 Residente de cirugía general III año Universidad Surcolombiana, Hospital Universitario Hernando Moncaleano Perdomo. Epidemiólogo. Neiva, Colombia.

3 Médico general, Universidad Surcolombiana. Neiva Colombia.

Correspondencia: Carlos Mauricio Martínez Montalvo. Correo: carlitos220792@gmail.com.

Fecha recibido: $\quad 08-08-17$

Fecha aceptado: 13-04-18

\begin{abstract}
Resumen
El megacolon tóxico (MT) es una patología con una tasa de mortalidad superior al $80 \%$ desencadenada por un proceso inflamatorio progresivo que compromete la pared del colon con dilatación secundaria de la luz intestinal debido a procesos inflamatorios o infecciosos. Su presentación clínica es infrecuente y los pilares básicos en su manejo son un diagnóstico oportuno, un manejo médico adecuado (antibiótico, reanimación hídrica y corrección metabólica) y, de ser necesario, un manejo quirúrgico eficaz que evite al máximo las complicaciones que empeoran el pronóstico de los pacientes. En este artículo se presenta un caso de una paciente con choque séptico secundario a MT, con desenlace fatal y con sospecha de un cuadro de colangitis grado III descartado por ecografía, lo cual generó distorsiones en su enfoque y manejo inicial. Por deterioro clínico y distensión abdominal, la paciente se llevó a laparoscopia diagnóstica en la que se evidenció un compromiso isquémico severo de todo el colon sin compromiso de intestino delgado, razón por la que se le realizó una colectomía total. El reporte de patología y la historia clínica descartan colitis ulcerativa o enfermedad de Crohn, lo que confirmó el MT. La paciente no presentaba factores de riesgo para el desarrollo de colitis pseudomembranosa. Se concluyó que fue la presentación de un caso de MT idiopático.
\end{abstract}

\section{Palabras clave}

Megacolon tóxico, colitis infecciosa, abdomen agudo, colitis, colangitis, sepsis.

\begin{abstract}
Toxic megacolon is a pathology whose mortality rate is over $80 \%$. A progressive inflammatory process compromises the colon wall, and secondary dilation of the intestinal lumen occurs due to inflammatory or infectious processes. Its clinical presentation is bizarre. but the basic pillars for management are opportune diagnosis and adequate medical management with antibiotics, water resuscitation, and metabolic correction. If necessary, effective surgical management can prevent the development of complications that worsen the disease and the prognosis of a patient. In this article we present the case of a patient who died after developing septic shock secondary to toxic megacolon. Cholangitis grade III was suspected, but discarded after ultrasonography, and this resulted in generated distortions in approach and initial management. Due to clinical deterioration and abdominal distension, the patient underwent diagnostic laparoscopy which revealed severe ischemic compromise of the entire colon but without involvement of the small intestine. For this reason, a total colectomy was performed. The pathology report and clinical history ruled out ulcerative colitis or Crohn's disease which confirmed the diagnosis of toxic megacolon. The patient had no risk factors for the development of pseudomembranous colitis. We conclude that this was a case of idiopathic toxic megacolon.
\end{abstract}

\section{Keywords}

Toxic megacolon, infectious colitis, acute abdomen, colitis, cholangitis, sepsis. 


\section{INTRODUCCIÓN}

El megacolon tóxico (MT), descrito por primera vez en 1950 como una complicación debida a un proceso infeccioso por Clostridium difficile, se caracteriza por un proceso inflamatorio progresivo que compromete las 4 capas del colon y se asocia con una dilatación $>6 \mathrm{~cm}$ segmental o total de la luz $(1,2)$. Su mortalidad oscila entre $19 \%$ a $80 \%$ en el peor de los casos y su incidencia varía dependiendo del factor causal: en colitis ulcerativa, de $2,5 \%$ a $17 \%$; y en colitis pseudomembranosa, de $0,4 \%$ a $3 \%$, valor en aumento debido al uso indiscriminado de terapia antibiótica (3-5).

La etiología clásica del MT es la colitis ulcerativa, pero la enfermedad de Crohn gradualmente ha tomado su papel desde 1950 después de descubrirse como un factor predisponente (6), así como otras etiologías tales como infecciones por Shigella, Salmonella, Entamoeba, Campylobacter, colitis isquémica, citomegalovirus (CMV) en inmunosuprimidos y sarcoma de Kaposi $(2,6)$.

Existen algunos factores de riesgo para el desarrollo de MT en pacientes con colitis infecciosa como la interrupción del tratamiento con esteroides, uso de enemas de bario y medicamentos que reducen la motilidad del colon tales como narcóticos, antidiarreicos y anticolinérgicos. La presentación clínica es muy infrecuente, por lo que se siguen aceptando los criterios clínicos descritos en 1969, que incluyen cualquiera de las siguientes condiciones: fiebre $>38,6$ ${ }^{\circ} \mathrm{C}\left(101,5^{\circ} \mathrm{F}\right)$; frecuencia cardíaca $(\mathrm{FC})>120$ latidos por minuto (lpm); leucocitos $>10,5 / \mu \mathrm{L}$; anemia (hemoglobina $[\mathrm{Hb}]<7 \mathrm{~g} / \mathrm{dL}$ ). También se asocia con uno de los siguientes criterios: deshidratación, hipotensión, alteraciones electrolíticas o cambios en el estado mental (7).

Los pilares del manejo del MT son la reanimación hídrica, corrección de electrólitos, administración de hemoderivados, manejo de terapia inmunosupresora y manejo antibiótico oportuno $(3,8,9)$. Las indicaciones absolutas para llevar a estos pacientes a tratamiento quirúrgico incluyen signos de insuficiencia orgánica, choque, hemorragia gastrointestinal baja incontrolable, evidencia de perforación, abdomen agudo y dilatación colónica progresiva después de 24 a 72 horas de tratamiento médico (10-12).

Se presenta el caso de una paciente con una sepsis de origen abdominal, inicialmente enfocada como un cuadro típico de colangitis, que se llevó a laparoscopia diagnóstica debido al deterioro clínico con evidencia de MT como hallazgo macroscópico, por lo que requirió colectomía total más ileostomía; posteriormente, este diagnóstico se confirmó con el reporte de patología. En esta oportunidad no se encontró una etiología clara que desencadenara el cuadro, por lo que este episodio de MT se clasificó como de un posible origen idiopático.

\section{CASO CLÍNICO}

La paciente era una mujer de 54 años de edad, con antecedentes de hipertensión arterial (HTA), hipotiroidismo y obesidad mórbida (índice de masa corporal [IMC]: 50,2) en manejo farmacológico con verapamilo, levotiroxina, ácido acetilsalicílico (ASA) y atorvastatina. Se presentó al servicio de urgencias de primer nivel por un cuadro clínico de 4 días de fiebre, dolor de hemiabdomen derecho irradiado a la región lumbar ipsilateral, astenia, adinamia y sensación de tremor. Los paraclínicos de primer nivel resultaron con un hallazgo de trombocitopenia, por lo que la remitieron a un nivel más avanzado debido a la sospecha de arbovirosis.

Ingresó al servicio de urgencias en condiciones generales regulares, polipneica, desaturada, somnolienta y febril con posterior insuficiencia ventilatoria, por lo que se inició una secuencia de intubación rápida sin complicaciones y se trasladó a la unidad de cuidado intensivo (UCI). Los paraclínicos de ingreso tuvieron hallazgos de trombocitopenia (70 000), acidosis metabólica con hiperlactatemia e injuria renal Acute Kidney Injury Network (AKIN) III. Se consideró que la paciente cursaba con choque séptico y posible foco en vías urinarias (pielonefritis), por lo cual se inició el cubrimiento antibiótico con piperacilina tazobactam, reanimación por metas, soporte inotrópico, manejo de comorbilidades y rastreo microbiológico.

$\mathrm{Su}$ evolución fue tórpida durante su estancia hospitalaria; la paciente presentaba oligoanuria, hipoperfusión distal, cifras tensionales limítrofes y se observaba un tinte ictérico en escleras. En los paraclínicos de control se evidenció leucocitosis en aumento con neutrofilia, trombocitopenia, acidosis metabólica moderada con anión gap elevado, transaminasas y amilasa normales, deterioro de la función renal, rayos $\mathrm{X}$ de tórax normales e hiperbilirrubinemia directa con patrón obstructivo, por lo que se solicitó una ecografía hepatobiliar por sospecha de cuadro de colangitis tipo III secundario a obstrucción de la vía biliar y se escalonó el manejo antibiótico con ertapenem. Debido al deterioro de su función renal, se valoró en el servicio de nefrología, que inició una terapia de reemplazo renal con hemodiálisis. Los resultados de hemocultivos solicitados reportaron hallazgos de Escherichia coli multisensible y urocultivo negativo (Tabla 1).

El reporte de ecografía no evidenció una litiasis vesicular ni dilatación de la vía biliar, por lo que se descartó un proceso séptico de origen biliar. Posteriormente, se evidenció en la paciente una distensión abdominal, por lo que se llevó por el servicio de cirugía general a laparoscopia diagnóstica en la que se evidenció líquido libre en la cavidad abdominal de predominio en gotera parietocólica derecha asociado con una marcada distensión del colon en su totalidad sin evidencia de procesos obstructivos mecánicos. El procedi- 
Tabla 1. Principales paraclínicos tomados durante la estancia hospitalaria

\begin{tabular}{|c|c|c|c|c|c|}
\hline Paraclínicos & 1 & 2 & 3 & 4 & 5 \\
\hline Leucocitos & 7000 & 9300 & 16400 & 28300 & 39400 \\
\hline Neutrófilos (\%) & 77 & 89 & 82,2 & 91,1 & 91 \\
\hline $\mathrm{Hb}$ & 12 & 12 & 10,3 & 9,4 & 8,6 \\
\hline Plaquetas & 69000 & 79000 & 49800 & 53000 & 41000 \\
\hline PCR & Negativa & & & 48 & \\
\hline TGO & & 64 & 89 & & 72 \\
\hline TGP & & 40 & 28 & & 44,6 \\
\hline PT/PTT/INR & & Normales & & Normales & Normales \\
\hline Sodio & & 134 & 135 & 137 & \\
\hline Potasio & & 3,7 & 4,31 & 4,6 & \\
\hline Cloro & & 102 & 104 & 107 & \\
\hline Calcio & & 1,01 & 1,04 & 1 & \\
\hline $\mathrm{Cr} / \mathrm{BUN}$ & $1,85 / 31$ & $1,55 / 36$ & $3,68 / 45,3$ & $4,32 / 55,8$ & $4,07 / 56,8$ \\
\hline Bilirrubina total & & 2,43 & 3,25 & 1,8 & 1,53 \\
\hline Bilirrubina directa & & 1,47 & 2,2 & 1,3 & 0,99 \\
\hline Fosfatasa alcalina & & 671 & & & 885 \\
\hline Amilasa & & 35 & & & 21 \\
\hline \multicolumn{6}{|l|}{ Gases arteriales } \\
\hline $\mathrm{pH}$ & & 7,16 & 7,22 & 7,20 & \\
\hline $\mathrm{PCO}_{2}$ & & 46 & 37 & 35 & \\
\hline $\mathrm{HCO}_{3}$ & & 16 & 15,4 & 13 & \\
\hline $\mathrm{PO}_{2}$ & & 145 & 145 & 153 & \\
\hline Lactato & & 4,1 & 1,7 & 1,6 & \\
\hline Base exceso & & -11 & -11 & $-13,3$ & \\
\hline $\mathrm{HBsAg}$ & & & & & Negativo \\
\hline VDRL & & & & & Negativo \\
\hline VIH & & & & & Negativo \\
\hline
\end{tabular}

BUN: nitrógeno ureico sanguíneo; Cr: creatinina; HBsAg: antígeno de la superficie de hepatitis $\mathrm{B}$; $\mathrm{HCO}_{3}$ : bicarbonato; INR: índice internacional normalizado; $\mathrm{PCO}_{2}$ : presión parcial de dióxido de carbono; PCR: proteína C-reactiva; $\mathrm{PO}_{2}$ : presión parcial de oxígeno; PT: tiempo de protrombina; PTT: tiempo parcial tromboplastina; TGO: transaminasa oxalacética; TGP: transaminasa pirúvica; VDRL: prueba Venereal Disease Research Laboratory; VIH: virus de la inmunodeficiencia humana.

miento se convirtió en laparotomía exploratoria por hallazgos de MT (Figura 1) y se realizó una colectomía total más ileostomía; además, se inició el cubrimiento antibiótico para C. difficile, Salmonella, Shigella y Campylobacter con ceftriaxona, vancomicina oral y metronidazol.

\section{REPORTE DE PATOLOGÍA}

El reporte de patología informó sobre el colon y segmento de íleon terminal, MT con dilatación luminal severa del ciego al sigmoides de hasta $22 \mathrm{~cm}$ de circunferencia y 107 $\mathrm{cm}$ de longitud, y aplanamiento de la mucosa y de las capas periféricas de la pared, sin cambios inflamatorios en la mucosa o en la pared que sugieran colitis ulcerativa o enfermedad de Crohn.

Por una parte, en su estadía hospitalaria postoperatoria se evidenció una mejoría clínica con disminución de soporte vasopresor, leucocitosis, aumento de gasto urinario con disminución de $\mathrm{Cr}$ y mejor patrón en gases arteriales; por otra parte, en la herida quirúrgica no se observaron signos de infección. En su octavo día postoperatorio sin soporte ventilatorio con estabilidad hemodinámica, se observó un cuadro de disnea súbita con evidencia de un paro cardiorrespiratorio; se activó el código azul con requerimiento de maniobras avanzadas de reanimación por 20 minutos sin tener éxito, por un cuadro clínico de disnea súbito en el 


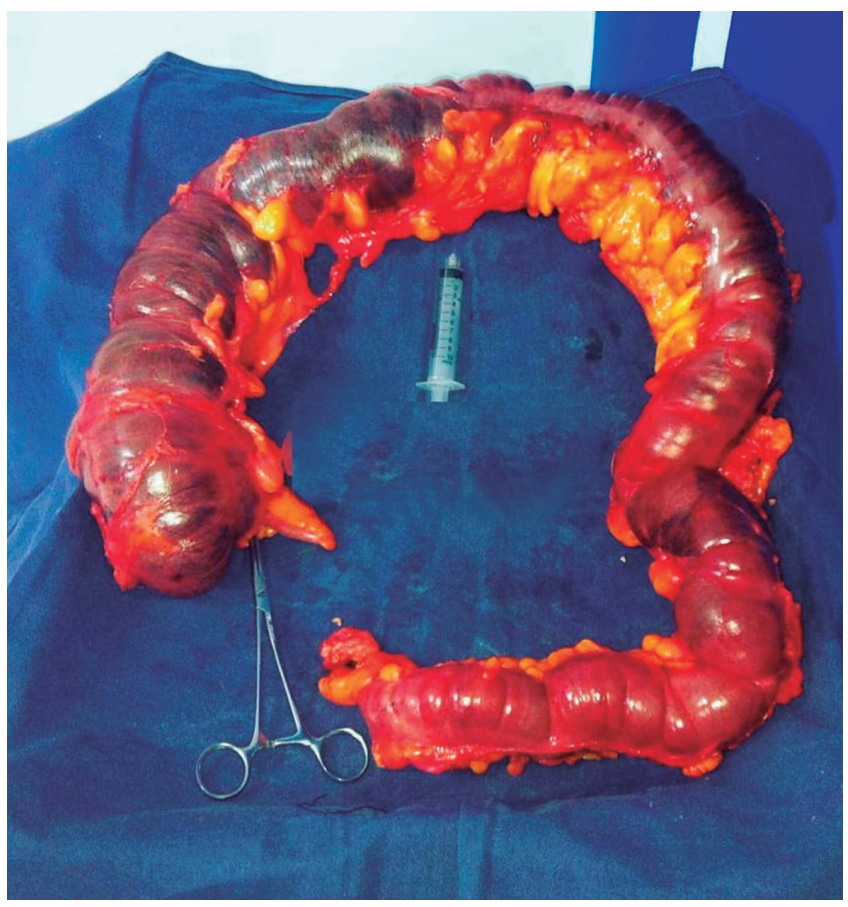

Figura 1. Colectomía total, sospecha de MT.

ámbito postoperatorio con un tromboembolismo pulmonar como posible causa de muerte.

\section{DISCUSIÓN}

El MT es una enfermedad letal con una incidencia general difícil de determinar con la literatura actual disponible; sin embargo, se ha logrado determinar que la incidencia se encuentra estrechamente relacionada con la causa en cuestión. Según algunos estudios, la colitis ulcerativa es un factor de riesgo 6 veces mayor para el desarrollo de MT que padecer la enfermedad de Crohn (3). En un estudio que incluyó 1236 pacientes admitidos a un hospital mostró una incidencia del $10 \%$ para estas 2 patologías (4).

En la actualidad, se considera una incidencia de MT secundario a colitis pseudomembranosa en aproximadamente $0,4 \%$ a $3 \%$ de casos; esta tasa se encuentra en aumento en la última década debido al uso de antibioticoterapia de manera deliberada, que ha causado cambios genéticos adaptativos de dicho microorganismo logrando mayores niveles de virulencia y, en consecuencia, el surgimiento de cepas resistentes a los tratamientos convencionales, como lo son el caso de las cepas BI/NAP1/027 (13-15).

El mecanismo fisiopatológico de esta enfermedad aún no se logra dilucidar totalmente; sin embargo, algunos estudios muestran un infiltrado severo y progresivo de la respuesta inflamatoria mediada por neutrófilos que logra comprometer desde la mucosa, pasando por la capa de musculo liso, hasta infiltrar la serosa. A medida que avanza la inflamación, los neutrófilos invaden la capa muscular y causan daño adicional por la liberación de enzimas proteolíticas, citocinas y leucotrieno $\mathrm{B}_{4}\left(\mathrm{LTB}_{4}\right)$, lo que resulta en dismotilidad $y$, en consecuencia, dilatación secundaria del colon (1) (Figura 2). En las causas infecciosas como la colitis pseudomembranosa, las toxinas A y B del C. difficile interrumpen la barrera epitelial y causan necrosis de células epiteliales y cambios electrofisiológicos en la mucosa colónica, cuyo resultado es una marcada inflamación del colon (8).

Es común que estos pacientes consulten los servicios de urgencias por un cuadro de deposiciones diarreicas (sanguinolentas) de más de una semana de evolución asociado con escalofríos, picos febriles y dolor abdominal como cólico intermitente; pero la aparición de MT es incoherente: en algunos casos se manifiesta por distensión abdominal, diarrea, estreñimiento, constipación, disminución de los ruidos intestinales y síntomas sistémicos como fiebre, taquicardia e hipotensión. Tales síntomas pueden ser enmascarados por altas dosis previas de corticosteroides o un nivel de conciencia alterado $(1,3,7)$. El diagnóstico del MT se realiza con base en la identificación del cuadro clínico y criterios clínicos descritos por Jalan en 1969 (16) asociado con toxicidad sistémica y con evidencia radiológica o ultrasonográfica de dilatación del colon $>6 \mathrm{~cm}(7,17)$.

Por una parte, la radiografía simple de abdomen y ecografía identifican el grado de dilatación del colon hasta de 15 $\mathrm{cm} \mathrm{y}>6 \mathrm{~cm}$, respectivamente (17); por otra parte, la tomografía axial computarizada (TAC) es útil para determinar la causa de complicaciones abdominales (7). Los hallazgos en laboratorios son la leucocitosis o leucopenia asociada con neutrofilia y anemia; alteraciones electrolíticas; y función renal, hepática y pulmonar. Se ha descrito que pacientes con leucocitosis $>40000$ presentan un factor de mal pronóstico (9). Es necesaria la toma de hemocultivos para descartar bacteriemia ya que la septicemia se produce hasta en el $25 \%$ de los pacientes con MT (3). Las muestras de heces deben ser enviadas para cultivo, sensibilidad y ensayo de toxina $\mathrm{A}$ y $\mathrm{B}$ de $C$. difficile en pacientes con antecedentes de uso de antibióticos o quimioterapia. Se debe considerar la infección de parásitos en pacientes con $\operatorname{VIH}(1,8)$.

Los componentes centrales del manejo incluyen resucitación con líquidos, corrección del desorden hidroelectrolítico, descompresión colónica (si es posible), administración de antibióticos y realización de la interconsulta pertinente al servicio de cirugía general. La anemia, la deshidratación y el déficit de electrólitos, en particular la hipopotasemia, agravan la dismotilidad del colon y deben tratarse agresivamente (18). La literatura recomienda la administración de 


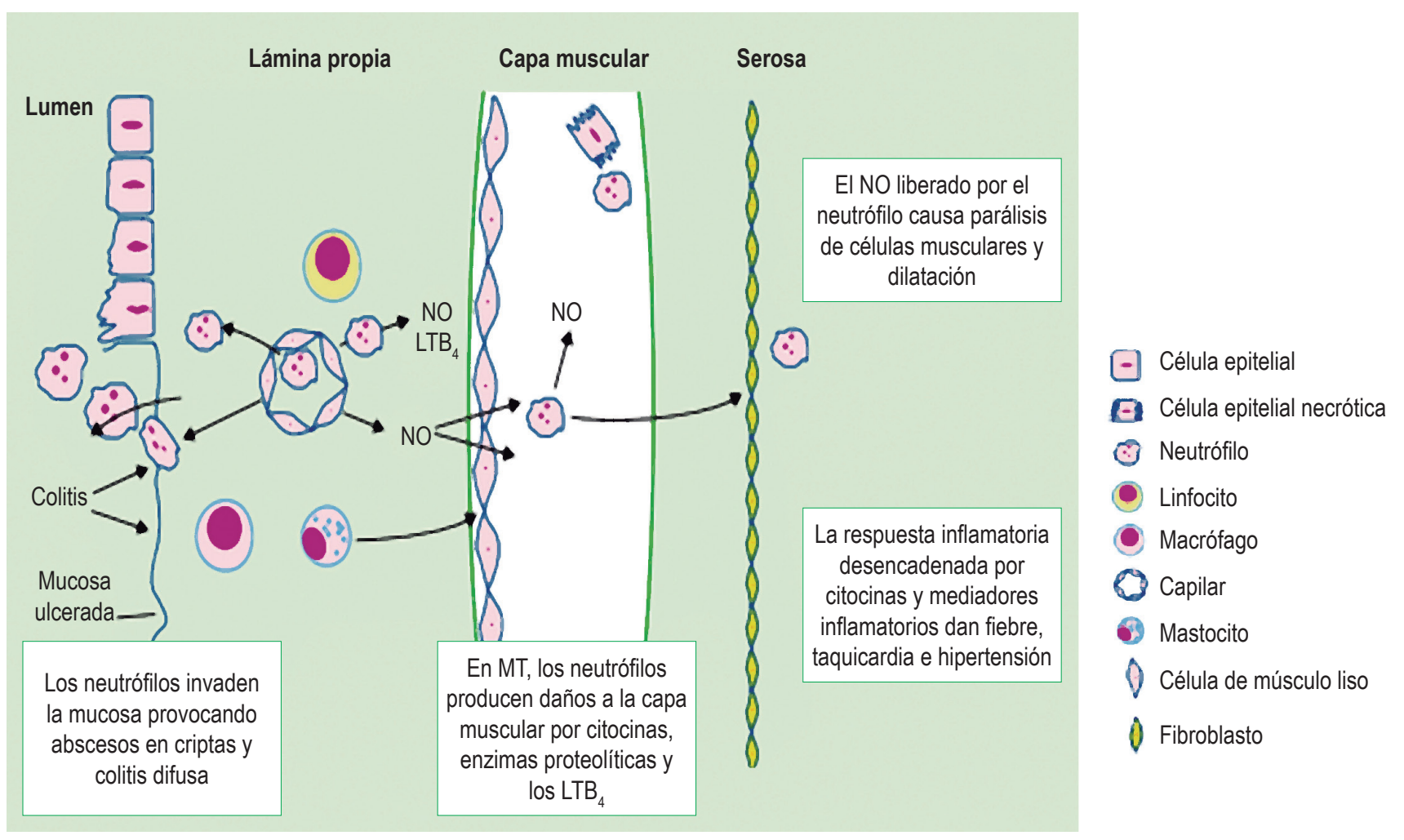

Figura 2. Representación gráfica de la invasión de los neutrófilos a través de todas las paredes del colon. NO: óxido nítrico. Tomado de: Sheth SG et al. Lancet. 1998;351(9101):509-13.

antibióticos de amplio espectro como la ampicilina, sulbactam o cefalosporina de tercera generación, asociados con un aminoglucósido o metronidazol $(9,19)$.

El pilar en el manejo médico de los pacientes con MT causado por colitis ulcerativa es el uso de esteroides intravenosos (IV) en altas dosis. La mayoría de los autores recomiendan una dosis diaria de $400 \mathrm{mg}$ de hidrocortisona (100 mg cada 6 horas) o $60 \mathrm{mg}$ de metilprednisolona (1 $\mathrm{mg} / \mathrm{kg}$ ) IV durante 5 días. Si este manejo no es efectivo en el paciente, se puede considerar la implementación de una terapia de rescate con ciclosporina $(8,19)$.

En los casos de MT causado por colitis pseudomembranosa, se deben identificar y retirar los antibióticos que pueden predisponer al cuadro clínico. Los antibióticos más comunes asociados con $C$. difficile son la clindamicina, las cefalosporinas y las fluoroquinolonas (20). La vancomicina asociada con metronidazol debe administrarse como terapia de primera línea de acuerdo con las directrices actuales emitidas por la Society for Healthcare Epidemiology of America y la Infectious Disease Society of America. En casos en los que se sospeche o confirme MT secundario a $C$. difficile resistente (cepas NAP1/BI/027), la fidaxomicina o un macrólido son los antibióticos de elección $(18,21)$.
La instauración de un tratamiento médico oportuno reduce en un 50\% la necesidad de un procedimiento quirúrgico (1); sin embargo, la intervención quirúrgica puede ser necesaria hasta en el $80 \%$ de los pacientes, principalmente en pacientes con MT secundario a $C$. difficile. El tratamiento quirúrgico de elección por gran parte de cirujanos en estos casos es la realización de colectomía subtotal más una fístula mucosa e ileostomía, por su relación con la reducción de morbimortalidad en los pacientes, en comparación con la realización de una proctocolectomía total. Es muy importante tener una valoración prioritaria por el servicio de cirugía general, ya que las complicaciones y, en mayor medida, la presencia de perforación intestinal generan un porcentaje exponencial de la mortalidad, pasando de un $8 \%$ hasta un $40 \%$ aproximadamente (1).

La morbimortalidad de los pacientes con MT es alta; se ha determinado que los pacientes que sobreviven a un episodio de MT con respuesta solo a tratamiento médico presentan un pobre pronóstico ( 6 a 12 meses) y presentan tasas de recurrencias superiores al $18 \%$, que pueden llegar a requerir colectomías. Entre los pacientes con colitis ulcerativa que inicialmente responden a la terapia médica, el $60 \%$ requerirá una colectomía en los siguientes 12 meses y 
el 80\% requerirá una colectomía dentro de los 5 años posteriores al primer cuadro (8).

En relación con el caso presentado, existe muy poca literatura referente a la aparición de casos de MT enmascarados por un cuadro de sospecha de colangitis aguda (22). Es importante recalcar que la paciente tenía factores de mal pronóstico (edad $>40$ años, hipoalbuminemia, insuficiencia renal y hiperlactatemia), que aumentaban exponencialmente su mortalidad; además, no se identificaron factores de riesgo para colitis pseudomembranosa. Después de la intervención quirúrgica, la paciente presentó una franca mejoría clínica; sin embargo, una entidad secundaria, posiblemente un tromboembolismo pulmonar, generó su deceso.

\section{CONCLUSIONES}

El MT es una complicación muy reconocida y altamente letal de la colitis aguda. En la actualidad, los médicos deben esperar un aumento en la incidencia de MT, debido al incremento de los casos de colitis agudas asociadas con el uso de antibióticos de amplio espectro y la coinfección de cepas de $C$. difficile hipervirulento e incluso resistente a las terapias convencionales.

Es esencial que los pacientes con MT sean diagnosticados rápida y correctamente, y manejados integralmente para disminuir la morbimortalidad. Los médicos de urgencias pueden minimizar los retrasos excesivos en el diagnóstico al sospechar y descartar esta entidad en todos los pacientes con distensión abdominal, diarrea aguda o crónica, y signos de respuesta inflamatoria sistémicos y, de este modo, mejorar el pronóstico de esta población.

\section{REFERENCIAS}

1. Sheth SG, LaMont JT. Toxic megacolon. Lancet. 1998;351(9101):509-13. doi: 10.1016/S01406736(97)10475-5.

2. Kwok M, Maurice A, Lisec C, et al. Campylobacter colitis: Rare cause of toxic megacolon. Intern J Surg Case Rep. 2016;27:141-3. doi: 10.1016/j.ijscr.2016.08.030.

3. Gan SI, Beck PL. A new look at toxic megacolon: an update and review of incidence, etiology, pathogenesis, and management. Am J Gastroenterol. 2003;98(11):2363-71. doi: 10.1111/j.1572-0241.2003.07696.x.

4. Greenstein AJ, Sachar DB, Gibas A, et al. Outcome of toxic dilatation in ulcerative and Crohn's colitis. J Clin Gastroenterol. 1985;7(2):137-43. doi: 10.1097/00004836198504000-00007.

5. Sayedy L, Kothari D, Richards RJ. Toxic megacolon associated Clostridium difficile colitis. World J Gastrointest Endosc. 2010;2(8):293-7. doi: 10.4253/wjge.v2.i8.293.

6. Meyers MA, Alonso DR, Morson BC, et al. Pathogenesis of diverticulitis complicating granulomatous colitis. Gastroenterology. 1978;74(1):24-31.
7. Earhart MM. The identification and treatment of toxic megacolon secondary to pseudomembranous colitis. Dimens Critical Care Nurs. 2008;27(6):249-54. doi: 10.1097/01. DCC.0000338869.70035.2b.

8. Halaweish I, Alam HB. surgical management of severe colitis in the intensive care unit. J Intensive Care Med. 2015;30(8):451-61. doi: 10.1177/0885066614534941.

9. Levine CD. Toxic megacolon: diagnosis and treatment challenges. AACN Clin Issues. 1999;10(4):492-9. doi: 10.1097/00044067-199911000-00008.

10. Fornaro R, Caratto M, Barbruni G, et al. Surgical and medical treatment in patients with acute severe ulcerative colitis. J Dig Dis. 2015;16(10):558-67. doi: 10.1111/17512980.12278.

11. Tapani MJ, Olavi KH. Surgical management of toxic megacolon. Hepatogastroenterology. 2014;61(131):638-41.

12. Teeuwen PH, Stommel MW, Bremers AJ, et al. Colectomy in patients with acute colitis: a systematic review. J Gastrointest Surg. 2009;13(4):676-86. doi: 10.1007/s11605-008-0792-4.

13. Dobson G, Hickey C, Trinder J. Clostridium difficile colitis causing toxic megacolon, severe sepsis and multiple organ dysfunction syndrome. Intensive Care Med. 2003;29(6):1030. doi: 10.1007/s00134-003-1754-7.

14. SynnottK, Mealy K, Merry C, et al. Timing of surgery for fulminating pseudomembranous colitis. BrJ Surg. 1998;85(2):22931. doi: 10.1046/j.1365-2168.1998.00519.x.

15. Zilberberg MD, Shorr AF, Kollef MH. Increase in adult clostridium difficile-related hospitalizations and casefatality rate, United States, 2000-2005. Emerg Infect Dis. 2008;14(6):929-31. doi: 10.3201/eid1406.071447.

16. Jalan KN, Sircus W, Card WI, et al. An experience of ulcerative colitis. I. Toxic dilation in 55 cases. Gastroenterology. 1969;57(1):68-82.

17. Maconi G, Sampietro GM, Ardizzone S, et al. Ultrasonographic detection of toxic megacolon in inflammatory bowel diseases. Dig Dis Sci. 2004;49(1):138-42. doi: 10.1023/B:DDAS.0000011615.64250.6e.

18. Leifeld L, Kruis W. Current management of toxic megacolon. Z Gastroenterol. 2012;50(3):316-22. doi: 10.1055/s0031-1299079.

19. Sobrado CW, Sobrado LF. Management of acute severe ulcerative colitis: a clinical update. Arq Bras Cir Dig. 2016;29(3):201-5. doi: 10.1590/0102-6720201600030017.

20. Yu S, Abdelkarim A, Nawras A, et al. Fecal transplant for treatment of toxic megacolon associated with Clostridium difficile colitis in a patient with duchenne muscular dystrophy. Am J Ther. 2016;23(2):e609-13. doi: 10.1097/ MJT.0000000000000062.

21. Hempel S, Newberry SJ, Maher AR, et al. Probiotics for the prevention and treatment of antibiotic-associated diarrhea: a systematic review and meta-analysis. JAMA. 2012;307(18):1959-69. doi: 10.1001/jama.2012.3507.

22. Juel J, Woyen AV, Vyberg M, et al. Primary manifestation of Chrohn's disease with toxic megacolon in a patient with long-time primary sclerosing cholangitis. Ugeskr Laeger. 2013;175(35):1965-6. 